\title{
Night shift work before and during pregnancy in relation to depression and anxiety in adolescent and young adult offspring
}

\author{
Susanne Strohmaier ${ }^{1,2} \cdot$ Elizabeth E. Devore $^{1} \cdot$ Celine Vetter $^{1,3} \cdot$ A. Heather Eliassen ${ }^{1,4} \cdot$ Bernard Rosner $^{1,5}$. \\ Olivia I. Okereke ${ }^{1,4,6} \cdot$ Eva S. Schernhammer ${ }^{1,2,4}$
}

Received: 19 July 2018 / Accepted: 2 May 2019 / Published online: 13 May 2019

(C) The Author(s) 2019

\begin{abstract}
We investigated the relationship between maternal history of nightshift work before and shift work during pregnancy and offspring risk of depression and anxiety, among mothers participating in the Nurses Health Study II and in their offspring enrolled in the Growing Up Today Study 2 between 2004 and 2013. Case definitions were based on offspring self-reports of physician/clinician-diagnosed depression and/or anxiety, regular antidepressant use and depressive symptoms assessed using the Center for Epidemiologic Studies Depression Scale. Multivariable-adjusted odds ratios (ORs) and 95\% confidence intervals (CIs) were estimated using generalized estimating equation models. We found no associations between maternal nightshift work before pregnancy or during pregnancy and offspring mental health disorders (e.g., nightshift work before pregnancy: depression (based on physician/clinician diagnosis): $\mathrm{OR}_{\text {ever nightwork }}=1.14 ; 95 \% \mathrm{CI}, 0.88-1.47$; either depression or anxiety: $\mathrm{OR}_{\text {ever nightwork }}=0.93 ; 95 \% \mathrm{CI}, 0.81-1.08$; nightshift work during pregnancy: depression: $\mathrm{OR}_{\text {ever nightwork }}=1.14$; 95\% CI, 0.68-1.94; depression or anxiety: $\mathrm{OR}_{\text {ever nightwork }}=1.17$; 95\% CI, 0.70-1.98) and no dose-response relationship with longer history of nightshift work (all $\mathrm{P}_{\text {Trend }}>0.10$ ). Stratifying by maternal chronotype revealed a higher risk of depression for offspring whose mothers worked nightshifts before pregnancy and reported being definite morning chronotypes (a proxy for circadian strain $)\left(\mathrm{OR}_{\text {ever nightwork }}=1.95 ; 95 \% \mathrm{CI}, 1.17,3.24\right.$ vs. $\mathrm{OR}_{\text {ever nightwork }}=0.93 ; 95 \% \mathrm{CI}, 0.68,1.28$ for any other chronotype; $\mathrm{P}_{\text {Interaction }}=0.03$ ). Further studies replicating our findings and refined understanding regarding the interplay of nightshift work and chronotype and its potential influences on offspring mental health are needed.
\end{abstract}

Keywords Circadian disruption $\cdot$ Intergenerational $\cdot$ Night shift work $\cdot$ Offspring mental health

Electronic supplementary material The online version of this article (https://doi.org/10.1007/s10654-019-00525-2) contains supplementary material, which is available to authorized users.

Eva S. Schernhammer

eva.schernhammer@meduniwien.ac.at

1 Channing Division of Network Medicine, Brigham and Women's Hospital and Harvard Medical School, Boston, MA, USA

2 Department of Epidemiology, Center for Public Health, Medical University of Vienna, Kinderspitalgasse 15, 1090 Vienna, Austria

3 Department of Integrative Physiology, University of Colorado, Boulder, CO, USA

4 Department of Epidemiology, Harvard T.H. Chan School of Public Health, Boston, MA, USA

5 Department of Biostatistics, Harvard T.H. Chan School of Public Health, Boston, MA, USA

6 Department of Psychiatry, Massachusetts General Hospital, and Harvard Medical School, Boston, MA, USA

\section{Introduction}

In the United States and worldwide, depression and anxiety are leading causes of morbidity and disability in young people; both negatively impact long-term health and pose a serious public health problem [1-3]. In 2015, the National Institute of Mental Health $[4,5]$ reported that $12.5 \%$ of the U.S. population aged 12 to 17 had experienced at least one major depressive episode in the past year and $10.3 \%$ of the population aged 18 to 25 . Further, a twelve-month prevalence of any mental illness (including depression and anxiety) of $21.7 \%$ was reported in that age group, following diagnostic criteria stated in the Diagnostic and Statistical Manual of Mental Disorder (DSM-IV) [6]. While prevalence estimates vary by country possibly due to different screening scales, clinical cut-points, or application of diagnostic criteria, the age-at-onset for most mental disorders is consistently reported with a median in the early- to mid-twenties across countries [7]. 
Established risk factors for depression and anxiety include individual-level characteristics (e.g., sex, age, psychosocial stress, substance use) and family-level characteristics (e.g., parental education, socio-economic status, family structure, family history) [8]. However, there is growing evidence that psychiatric disorders may have developmental origins, and maternal exposure may impact offspring mental health via fetal programming [9, 10].

Work schedules involving rotating night shift work have previously been linked to mental disorders [11-15] and other chronic diseases such as type 2 diabetes [16], cardiovascular diseases [17] and several cancers [18, 19], with some evidence that inter-individual differences in chronotype could modify these relationships [19, 20]. Moreover, recent studies suggest that shift working nurses experience epigenetic alterations (e.g., methylation patterns in the promoter region of the serotonin transporter gene) [21,22], which could contribute to development of depressed mood in offspring [23].

Additionally, night shift work surrounding pregnancy, through regulation of the maternal melatonin profile, may lead to behavioral programming in the offspring via alterations of the hypothalamic-pituitary-adrenal (HPA) axis [24, 25]. More specifically, melatonin can freely cross the placenta and provide photoperiodic information to the fetus [26] and in turn influence the development of the circadian system [27]. Evidence form animal models shows that maternal melatonin is involved in the development of the fetal adrenal gland and glucocorticoid signaling [28] which are reportedly important in the programming of emotional behaviors [29].

Animal models also revealed that parental social defeat stress prior to conception can lead to depression and anxiety behaviors in offspring rodents [30]. In humans, so far only extreme stressors prior to conception or during pregnancy (e.g., Holocaust, World Trade Center attack) have been linked to adverse mental outcomes in offspring [31-33].

However, no prior study has examined the association of night shift work (an established health stressor [34]) before and during pregnancy, maternal differences in preferred wake/sleep time (i.e., chronotype), and offspring mental health. To provide insights regarding these associations, we combined existing shift work information in $>4000$ women participating in the Nurses' Health Study II with information on lifetime risk of depression and anxiety in their offspring, who were enrolled in the Growing Up Today Study 2.

\section{Methods}

\section{Study population}

Our present analyses included mother-child pairs, comprised of mothers who were participants in the prospective Nurses' Health Study II (NHSII) and their children who were participants in the Growing Up Today Study 2 (GUTS2). NHSII is an ongoing prospective cohort study. It was established in 1989, when 116,429 US female nurses aged 25-42 years responded to a mailed questionnaire about their health and lifestyle; biennial follow-up questionnaires are used to update information on risk factors and medical history.

GUTS2 was established in 2004, when after obtaining maternal consent, invitation letters and questionnaires were sent to 17,280 children aged 9 to 15 years, born to NHSII participants between 1987 and 1995. Of these, 10,918 children returned completed questionnaires and information on health and lifestyle factors with updated information from follow-up questionnaires in 2006, 2008, 2011, and 2013.

This study has approval from the Committees on the Use of Human Subjects in Research at the Brigham and Women's Hospital and the Harvard T.H. Chan School of Public Health (Boston, MA, USA). If participants returned the baseline self-administered questionnaire, it was assumed to imply informed consent in both cohorts.

\section{Ascertainment of night shift work among mothers}

\section{Mother's history of rotating night shift work before pregnancy}

Information on the history of rotating night shift work was first obtained in the NHSII baseline questionnaire in 1989, when nurses were asked to report their total years of rotating night shift work, defined as "at least three nights per month in addition to working days or evenings in the respective month". Using updated information from questionnaires in 1991 and 1993 and retrospective assessments for the period between 1993 and 1995 from the questionnaire in 2001, cumulative shift work exposure was derived by adding together the number of years participating in shift work before conception, for children born between 1989 and 1995 .

\section{Mother's night shift work exposure during pregnancy}

Additional information regarding specific occupational exposures, including shift work during the most recent pregnancy since 1993, was collected through a supplemental questionnaire following the main NHSII questionnaire in 2001. Participants who had previously reported at least one pregnancy since 1993, worked as a nurse and indicated their willingness to participate were sent additional questions that asked about working schedules and number of night shifts per month for each trimester, along with other exposures. 
Timeline of exposure and outcome assessment

GUTS 2 (birth years 1987-1995)

$200420062008 \quad 20112013$

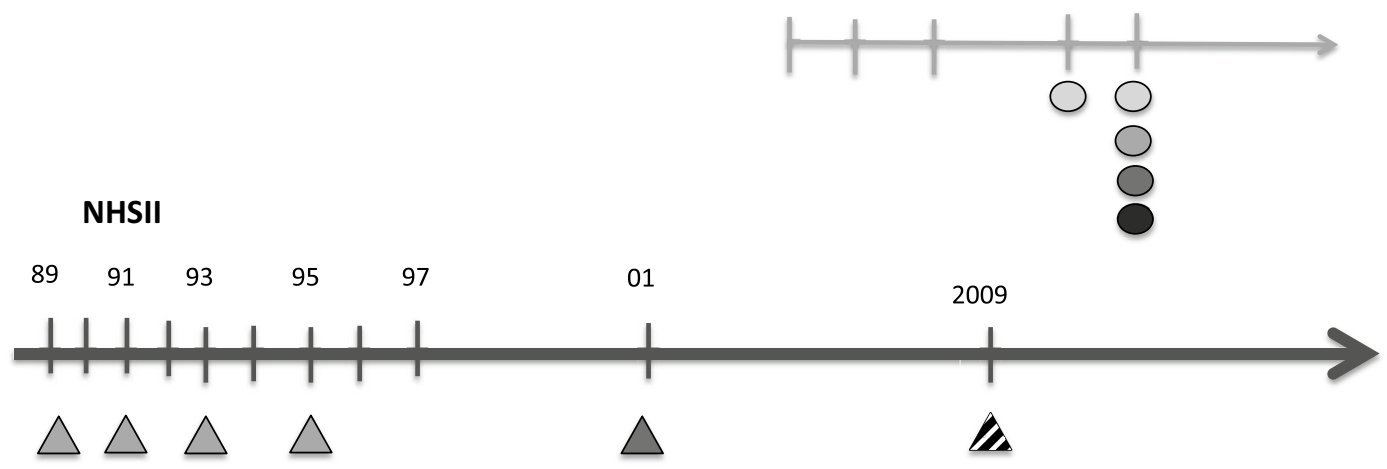

Exposures

History of night shift work (total number of years in 1989, updates for the past 24 months thereafter

Pregnancy questionnaire, asking about night work during pregnancy for the most recent pregnancy since 1993

Maternal chronotype assessment

\section{Outcomes}

Depressive symptoms assessment using CESD-10

Self-reports of physician/clinician

diagnosed depression

Self-reports of SSRI use

Self-reports of physician/clinician diagnosed anxiety

Fig. 1 Timeline of exposure assessment in NHSII and outcome assessment in GUTS2

\section{Ascertainment of mental health disorders among offspring}

In this study, we utilized multiple sources of information on depression. First, we defined depression cases through self-report of physician/clinician-diagnosed depression, which was collected on the 2013 GUTS questionnaire. At that time, participants were also asked to indicate the year of first diagnosis (before 2006, 2006-2008, 2009-2011, 2012 or later). An alternative, broader Boolean OR case definition was based on reporting a physician/clinician-diagnosed depression, regular antidepressant use, or the presence of clinically significant depressive symptoms [35]. Information on selective serotonin reuptake inhibitors (SSRIs) use was obtained from the 2013 GUTS questionnaire, when participants were asked about regular medication use in the past 12 months. Depressive symptoms were assessed using the Center for Epidemiological Studies Depression Scale10 (CESD-10) [36, 37] in 2011 and 2013. The presence of clinically significant depressive symptoms was defined as having a CESD-10 score $\geq 10$ in either 2011 or 2013 [38]; the CESD-10 $\geq 10$ cutoff has been previously validated for major depression. Cases of anxiety were defined based on self-report of physician/clinician-diagnosed anxiety on the 2013 GUTS questionnaire. A composite psychiatric outcome was created, including participants as cases if they: selfreported diagnosis of depression or anxiety, used antidepressant medication, and/or scored $\geq 10$ points on the CESD-10 at any time. A timeline of exposure and outcome assessments is shown in Fig. 1.

\section{Ascertainment of covariates}

To calculate maternal age at delivery, we computed the difference between mother's and child's dates of birth. Maternal diet quality was characterized based on the validated food frequency questionnaire (FFQ) [39] from the 1991 main NHSII questionnaire, from which the Alternative Healthy Eating Index (AHEI) and alcohol intake were derived [40]. In 1989, participants were asked to report frequency of various physical activities, which was used to derive energy expenditure in metabolic equivalent (MET) hours per week [41]. Information on smoking habits and weight and height was collected biennially; this was applied in determining the status of smoking and anthropomorphic measures that were most proximal to and before conception of the first included child. Maternal diagnosis of diabetes and high blood pressure before conception were ascertained on the NHSII baseline questionnaire in 1989, and maternal lifetime history of depression was selfreported in NHSII beginning in 2003. Maternal chronotype 
was assessed in 2009 using a single question from the Morningness-Eveningness Questionnaire [42], which has been validated for classifying individuals as "definitely a morning type", "rather more a morning than an evening type", "rather more an evening than a morning type", "definitely an evening type" or neither.

To capture the family's socioeconomic status surrounding a child's birth and early childhood, we used US Census tract data [43] from the 1989 U.S. Census. We considered the variables median income and percentage of the population holding a college degree in the mother's neighborhood, which could be extracted based on the mother's address at that time. Additionally, we ascertained information on husbands' level of education in 1999, mother's subjective social and economic standing in her community and the US, and mother's adulthood household income reported in 2001. Further, we considered information on the geographic region children were living at the GUTS2 baseline in 2004.

The GUTS2 baseline questionnaire also assessed offspring stage of pubertal development (Tanner stage), using a validated scale of pubic hair illustrations [44]. Further, offspring sedentary behavior was derived by summing up the reported time they had spent watching television, using the computer, surfing on the internet, and reading/doing homework.

\section{Final samples for analyses}

Since history of maternal rotating night shift was assessed for the first time in 1989, we only considered children born in 1989 or later in our analyses; hence, we excluded 4721 of the original cohort of 10,918 children. Further, we excluded twins and triplets (195 children, 87 mothers), children born at less than 37 weeks gestation (i.e., non full-term pregnancies) (1183 children, 1001 mothers), and mother-child pairs with missing exposure information (7 children, 6 mothers). In total, we included 4813 children born to 4044 mothers in our analyses of pre-conception shift work and mental health outcomes in offspring.

To assess the associations between night shift work during pregnancy and depression and anxiety in offspring, we identified a subset of 621 GUTS2 participants whose mothers participated in the occupational supplemental questionnaire. After excluding children born at less than 37 weeks gestation (72 children, 72 mothers) and pairs with missing exposure information ( 4 children, 4 mothers), a total of 545 mother-child pairs were left for these analyses.

\section{Statistical analysis}

\section{Mother's history of rotating night shift exposure before conception}

To account for clustering within families, we used generalized estimating equation (GEE) regression models specifying a logit link function and an exchangeable correlation structure to estimate odds ratios (ORs) and $95 \%$ confidence intervals (CIs) for depression and anxiety outcomes in offspring across four categories of cumulative rotating night shift work before conception (none, $<3$ years, $3-5$ years, and $\geq 6$ years). Mothers without a history of night shift work were considered the reference group. We also used this approach to examine outcomes in offspring comparing mothers who ever worked night shifts versus mothers who never worked night shifts.

Initial models were adjusted for offspring baseline age and sex (basic model). We further considered a wide range of covariates describing maternal lifestyle and social economic status, including BMI and smoking status before pregnancy, AHEI dietary score, physical activity, husband's education, geographic region at GUTS baseline, and the Census tract education rate in 1989 in multivariate model 1 (MV model 1). Lastly, maternal lifetime history of depression was added to the covariates included in MV model 1 (MV model 2). We included a missing indicator in our models when covariates had missing values.

\section{Mother's night shift work exposure during pregnancy}

Based on the information provided in the supplemental questionnaire, we used logistic regression models to estimate odds ratios and $95 \%$ CIs for offspring depression and anxiety comparing children born to mothers who worked night shifts during pregnancy to those whose mothers did not. Covariates were added to the models following a similar selection procedure as described above.

Previous studies have identified associations between chronotype and mental health outcomes [45, 46], and effect modification of associations between night shift work and chronic diseases by chronotype $[19,20]$. Therefore, we assessed possible effect modification of the association between night shift work and offspring mental health outcomes by maternal chronotype. To test for effect modification, we included multiplicative interaction terms in multivariable regression models.

All analyses were conducted using SAS version 9.4 (SAS Institute, Cary, NC) and all statistical tests were twosided and considered statistically significant at $P<0.05$. 


\section{Results}

\section{Mother's history of rotating night shift work before pregnancy}

The 4044 mothers included in our analyses were, on average, 33.4 (SD 3.6) years old when they delivered their children in this study, while the 4813 children were on average 11.7 (SD 1.2) years old when they started participating in GUTS2. There were more girls than boys included in the study (53.4\% girls, $46.6 \%$ boys). Comparing maternal and offspring characteristics across categories of duration of night shift work before conception (65\% of the mothers had reported night shift work), we found only modest differences (Table 1). Mothers reporting longer durations of night shift work were slightly older at birth, and more likely to be past or current smokers and to adhere to a healthy diet (AHEI); they also reported a slightly higher BMI before pregnancy and a higher frequency of lifetime physician/clinician-diagnosed depression.

We did not observe an overall association between maternal night shift work before pregnancy and risk of any of depression and anxiety in their offspring (Table 2). However, there was some indication that maternal chronotype might play a role in the relationship between maternal night shift work before pregnancy and depression in offspring (Table 3). Because differences between basic and multivariable adjusted models were small, we focus on the results obtained from fully-adjusted models only (MV models 2 adjusting for the largest set of relevant covariates).

We found no association between maternal night shift work and offspring depression, regardless of the case definition that was applied: e.g. risk of physician/clinician-diagnosed depression $\left(\mathrm{OR}_{\text {ever nightwork }}=1.14 ; 95 \%\right.$ CI, $0.88,1.47$ ); risk of depression using the broader case definition (physician-diagnosed depression or SSRI use or CESD $10 \geq 10$ ); $\mathrm{OR}_{\text {ever nightwork }}=0.94 ; 95 \% \mathrm{CI}, 0.81,1.09$ ) (Table 2). Also, children born to mothers who had worked night shifts had a similar risk of physician-diagnosed anxiety $\left(\mathrm{OR}_{\text {ever nightwork }}=0.85 ; 95 \% \mathrm{CI}, 0.66,1.11\right)$ and similar risk of any outcome $\left(\mathrm{OR}_{\text {ever nightwork }}=0.93 ; 95 \% \mathrm{CI}, 0.81\right.$, 1.08), compared to children born to mothers without shiftwork history. Similarly, duration of night shift work history was not associated with risk of any of the outcomes considered (all $\mathrm{P}_{\text {Trend }}>0.10$ ) (Table 2).

We conducted analyses stratified by maternal chronotype (Table 3). Compared to offspring of women without a history of rotating night shift work, offspring of women with any rotating night work before pregnancy had a significantly elevated risk of physician-diagnosed depression among women who were definite morning chronotypes
$\left(\mathrm{OR}_{\text {ever nightwork }}=1.95 ; 95 \% \mathrm{CI}, 1.17,3.24\right)$, whereas this was not the case for offspring of women with intermediate or evening chronotypes $\left(\mathrm{OR}_{\text {ever nightwork }}=0.93 ; 95 \%\right.$ CI, 0.68, 1.28; $\left.\mathrm{P}_{\text {Interaction }}=0.03\right)$. Similar indications of effect modification were not observed for the alternative-Boolean OR - case definition of depression (based on reporting a physician/clinician-diagnosed depression, regular antidepressant use, or the presence of clinically significant depressive symptom) or the other considered outcomes $\left(\mathrm{P}_{\text {interaction }}\right.$ for broader depression definition $=0.96$, for self-reported anxiety $=0.24$, for any psychological outcome $=0.96$ ).

\section{Maternal night shift work exposure during pregnancy}

A detailed description of maternal and offspring characteristics for the subsample with available exposure information during pregnancy can be found elsewhere [47].

We restricted our analyses to the broad endpoints of any depression or anxiety outcome, as well as the depression definition based on physician-diagnosed depression, SSRI use, or depressive symptoms (CESD-10 $\geq 10$ ). Again, results from basic and multivariable models did not differ markedly (Supplemental Table 1), so we focus on results from the fully-adjusted models (MV model 2) only. Briefly, risks of depression and anxiety for children of women with or without night shift work exposure during pregnancy were not significantly different (risk of depression $\mathrm{OR}_{\text {ever nightwork }}=1.14$; 95\% CI, 0.68, 1.94; risk of any psychiatric outcome $\left.\mathrm{OR}_{\text {ever nightwork }}=1.17 ; 95 \% \mathrm{CI}, 0.70,1.98\right)$.

\section{Discussion}

In this study including mothers enrolled in NHSII and their offspring participating in GUTS2, we found no overall associations of maternal history of rotating night shift work before pregnancy or shift work exposure during pregnancy with depression or anxiety in their children. Among mothers who were definite morning chronotypes, there was significantly higher risk of physician/clinician-diagnosed depression offspring of mothers who participated in rotating night work before pregnancy compared to offspring of mothers who did not. Hence, there is some indication that biologically-based maternal preferences in sleep timing might play a role in the relationship between maternal night shift work before pregnancy and depression outcomes, suggesting that social and biological stress induced by an extreme mismatch of preferred and actual sleeping times might have an impact on offspring mental health. Interestingly, we identified this effect modification only for self-reported physician/clinician-diagnosed depression and not for any of the 


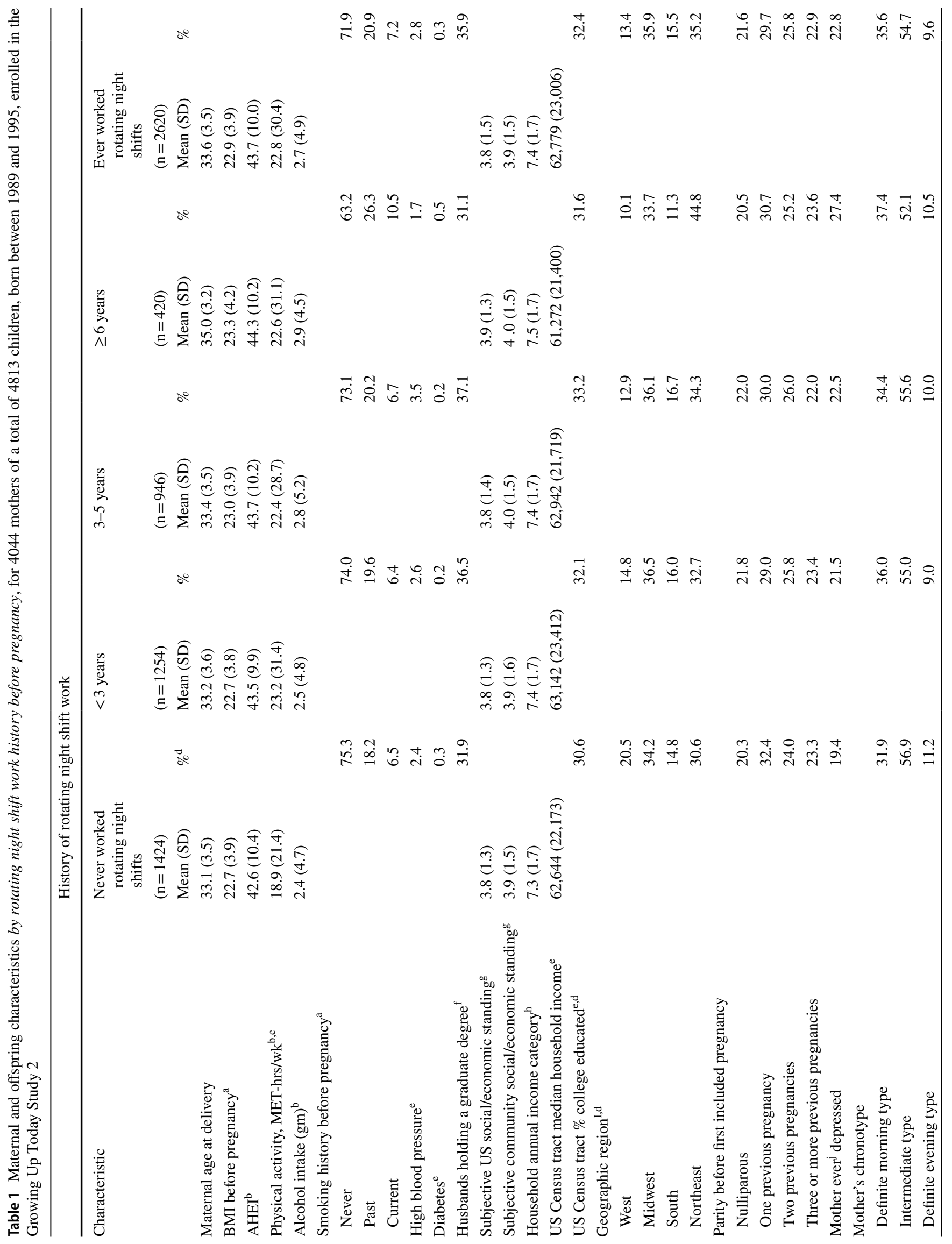




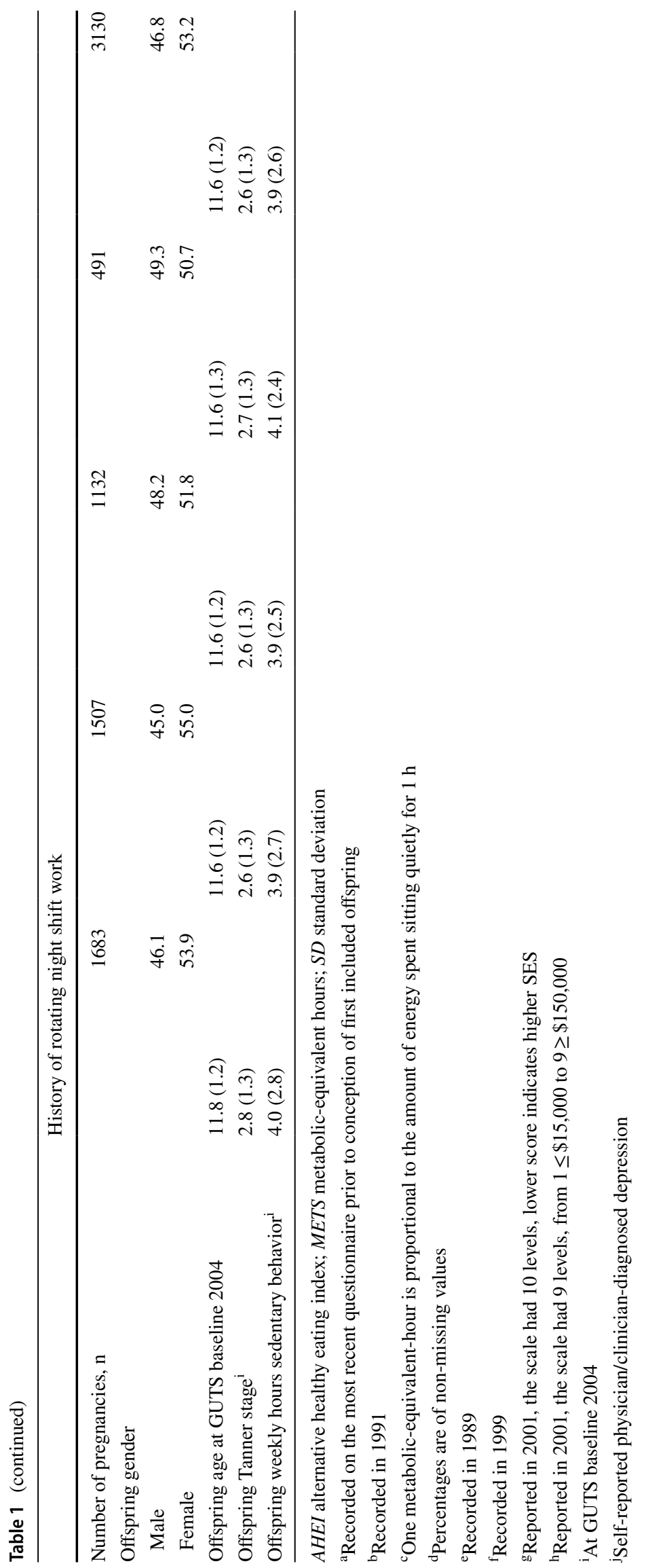


Table 2 Adjusted odds ratios (OR) for offspring risk of depression during childhood and adolescence according to maternal rotating night shiftwork history before pregnancy, using data from the Growing Up Today Study 2 from 2004 to 2013, restricted to singleton, full-term births

\begin{tabular}{|c|c|c|c|c|c|c|c|c|c|c|}
\hline & \multicolumn{10}{|c|}{ History of rotating night shift work } \\
\hline & $\begin{array}{l}\text { Never worked } \\
\text { rotating night } \\
\text { shifts }\end{array}$ & \multicolumn{2}{|l|}{$<3$ years } & \multicolumn{2}{|l|}{$3-5$ years } & \multicolumn{3}{|c|}{$\geq 6$ years } & \multicolumn{2}{|c|}{$\begin{array}{l}\text { Ever worked rotat- } \\
\text { ing night shifts }\end{array}$} \\
\hline \multicolumn{11}{|c|}{ Self-reported physician/clinician—diagnosed depression* } \\
\hline \multirow[t]{2}{*}{ Cases/participants } & $92 / 1683$ & \multicolumn{2}{|c|}{$99 / 1507$} & \multicolumn{2}{|l|}{$78 / 1132$} & \multicolumn{2}{|l|}{$23 / 491$} & & \multicolumn{2}{|c|}{$200 / 3130$} \\
\hline & OR & OR & $95 \% \mathrm{CI}$ & OR & $95 \% \mathrm{CI}$ & OR & $95 \% \mathrm{CI}$ & $P$ trend & OR & $95 \% \mathrm{CI}$ \\
\hline Basic model $^{\mathrm{a}}$ & 1 (reference) & 1.25 & $0.93,1.68$ & 1.33 & $0.97,1.82$ & 0.88 & $0.55,1.40$ & 0.63 & 1.22 & $0.94,1.57$ \\
\hline MV model $1^{\mathrm{b}}$ & 1 (reference) & 1.20 & $0.89,1.62$ & 1.26 & $0.92,1.73$ & 0.82 & $0.51,1.33$ & 0.82 & 1.17 & $0.90,1.51$ \\
\hline MV model $2^{\mathrm{c}}$ & 1 (reference) & 1.18 & $0.87,1.60$ & 1.23 & $0.89,1.69$ & 0.79 & $0.49,1.27$ & 0.99 & 1.14 & $0.88,1.47$ \\
\hline \multicolumn{11}{|c|}{ Self-reported physician/clinician—diagnosed depression* or SSRI** use or CESD10 $\geq 10^{x}$} \\
\hline \multirow[t]{2}{*}{ Cases/participants } & $389 / 1683$ & \multicolumn{2}{|c|}{$346 / 1507$} & \multicolumn{2}{|c|}{$263 / 1132$} & \multicolumn{2}{|l|}{$91 / 491$} & & \multicolumn{2}{|c|}{$700 / 3130$} \\
\hline & OR & OR & $95 \% \mathrm{CI}$ & OR & $95 \% \mathrm{CI}$ & OR & $95 \% \mathrm{CI}$ & $\mathrm{P}$ trend & OR & $95 \% \mathrm{CI}$ \\
\hline Basic model $^{\mathrm{a}}$ & 1 (reference) & 0.99 & $0.84,1.17$ & 1.02 & $0.85,1.23$ & 0.77 & $0.59,0.99$ & 0.25 & 0.96 & $0.83,1.11$ \\
\hline MV model $1^{\mathrm{b}}$ & 1 (reference) & 0.98 & $0.83,1.16$ & 1.00 & $0.83,1.20$ & 0.74 & $0.56,0.96$ & 0.16 & 0.95 & $0.82,1.10$ \\
\hline MV model $2^{\mathrm{c}}$ & 1 (reference) & 0.97 & $0.82,1.15$ & 0.99 & $0.82,1.19$ & 0.73 & $0.56,0.95$ & 0.13 & 0.94 & $0.81,1.09$ \\
\hline \multicolumn{11}{|c|}{ Self-reported physician/clinician—diagnosed anxiety* } \\
\hline \multirow[t]{2}{*}{ Cases/participants } & $98 / 1683$ & \multicolumn{2}{|l|}{$80 / 1507$} & \multicolumn{2}{|l|}{$60 / 1132$} & \multicolumn{2}{|l|}{$25 / 491$} & & \multicolumn{2}{|c|}{$165 / 3130$} \\
\hline & OR & OR & $95 \% \mathrm{CI}$ & OR & $95 \% \mathrm{CI}$ & OR & $95 \% \mathrm{CI}$ & $P$ trend & OR & $95 \% \mathrm{CI}$ \\
\hline Basic model $^{\mathrm{a}}$ & 1 (reference) & 0.92 & $0.68,1.25$ & 0.94 & $0.68,1.32$ & 0.91 & $0.58,1.43$ & 0.63 & 0.93 & $0.71,1.20$ \\
\hline MV model $1^{\mathrm{b}}$ & 1 (reference) & 0.88 & $0.64,1.20$ & 0.90 & $0.64,1.25$ & 0.82 & $0.52,1.31$ & 0.43 & 0.88 & $0.67,1.14$ \\
\hline MV model $2^{\mathrm{c}}$ & 1 (reference) & 0.86 & $0.63,1.18$ & 0.86 & $0.61,1.21$ & 0.79 & $0.50,1.26$ & 0.30 & 0.85 & $0.66,1.11$ \\
\hline \multicolumn{11}{|c|}{ Combination of any psychiatric outcome ${ }^{\S}$} \\
\hline \multirow[t]{2}{*}{ Cases/participants } & $407 / 1683$ & $361 / 1507$ & & $272 / 1132$ & & $96 / 491$ & & & $729 / 3$ & \\
\hline & OR & OR & $95 \% \mathrm{CI}$ & OR & $95 \% \mathrm{CI}$ & OR & $95 \% \mathrm{CI}$ & $\mathrm{P}$ trend & OR & $95 \% \mathrm{CI}$ \\
\hline Basic model $^{\mathrm{a}}$ & 1 (reference) & 0.99 & $0.84,1.17$ & 1.01 & $0.84,1.21$ & 0.77 & $0.60,1.00$ & 0.22 & 0.96 & $0.83,1.11$ \\
\hline MV model $1^{\mathrm{b}}$ & 1 (reference) & 0.97 & $0.82,1.15$ & 0.99 & $0.82,1.19$ & 0.74 & $0.57,0.96$ & 0.14 & 0.94 & $0.82,1.09$ \\
\hline MV model $2^{\mathrm{c}}$ & 1 (reference) & 0.97 & $0.82,1.14$ & 0.98 & $0.81,1.17$ & 0.73 & $0.56,0.95$ & 0.10 & 0.93 & $0.81,1.08$ \\
\hline
\end{tabular}

CESD10 Center for Epidemiologic Studies Depression Scale; $C I$ confidence interval; $M V$ multivariable model; $O R$ odds ratio

*Assessed in 2013 (timing: before 2005; between 2006 and 2008; between 2009 and 2011, 2012+)

**Assessed in 2013 (intake in the past 12 months)

${ }^{\mathrm{x}}$ Assessed in 2011 and 2013

${ }^{\S}$ Defined as self-reported physician/clinician—diagnosed depression * OR SSRI ** use OR CESD10 $\geq 10$ OR self-reported physician/clinician— diagnosed anxiety

${ }^{a}$ Adjusted for offspring gender (boy/girl) and offspring age at GUTS baseline 2004

${ }^{b}$ Additionally adjusted for maternal age at pregnancy, smoking status before pregnancy (never, current, past), alternative healthy eating score (quintiles), physical activity (METs hours/week; quintiles), husband's education (less than 2 year college, 4 year college, grad school), parity (nulliparity, 1, 2, 3+ previous pregnancies), BMI before pregnancy $\left(<25,25-29, \geq 30 \mathrm{~kg} / \mathrm{m}^{2}\right)$, geographic region (West, Midwest (reference), South, Northeast) and Census tract education rate in 1989

${ }^{\mathrm{c}}$ Additionally adjusted for maternal depression diagnosis (yes/no)

other considered outcomes, including a broader depression definition that also included antidepressant use and depressive symptoms. Possible explanations are that self-reported physician/clinician-diagnosed depression may capture more severe depression cases, may have higher specificity and precision than the broad definition, or may indicate earlier age-at-onset cases.

Rotating night shift work, an established social and biological stressor [34, 48], and chronotype have independently been linked to increased risk of depression $[11,12,14,45,49,50]$, and an interaction of chronotype and work hours has been reported previously for chronic disease outcomes $[19,20]$ within the same person. Growing literature, including animal and human studies, supports the hypothesis that prenatal programming can impact psychiatric disorders, which may be driven by alterations of the HPA axis and, in turn, effects on the circadian and limbic system [10]. Nevertheless, to the best of our 


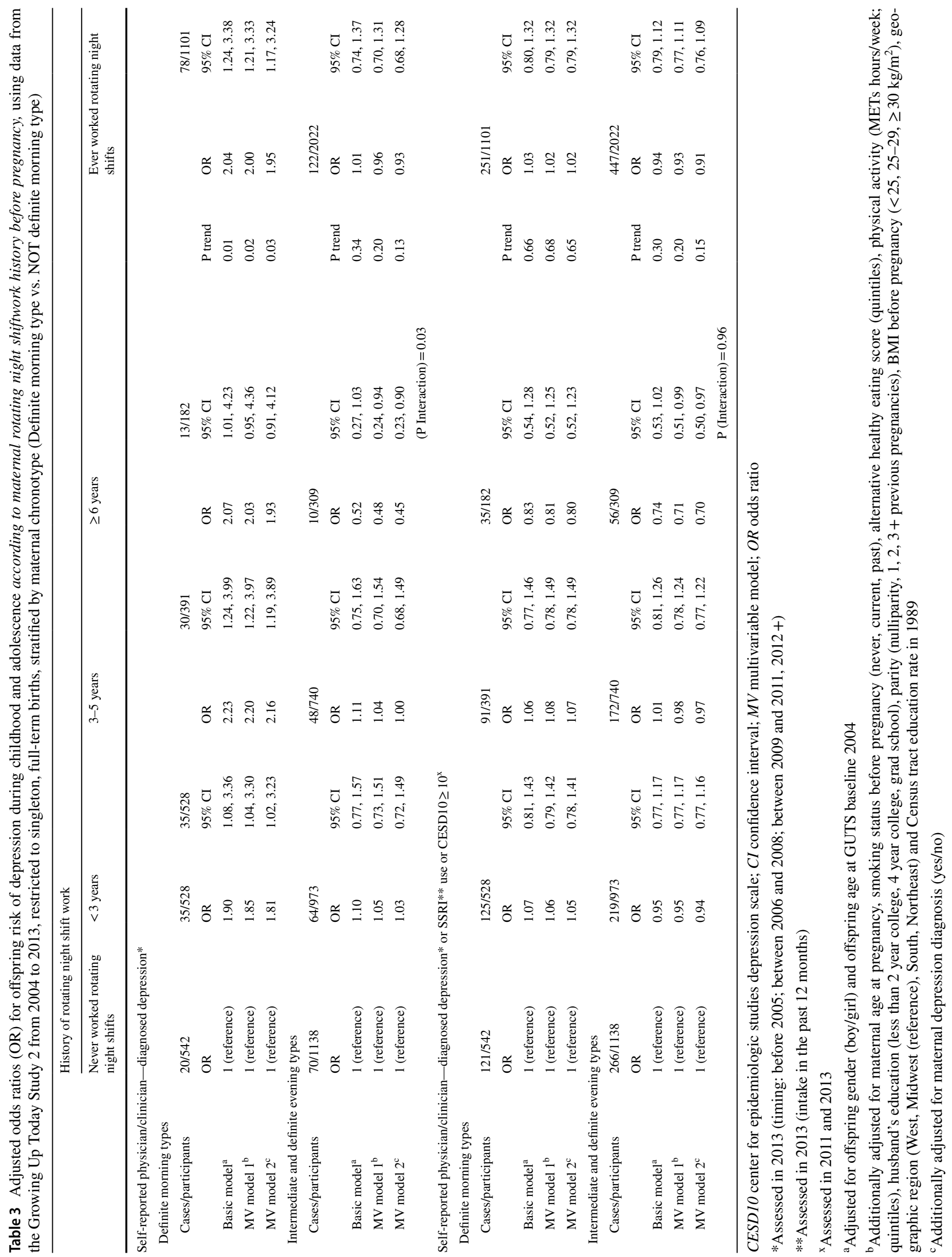


knowledge, there is no study so far that investigated the intergenerational associations of maternal night shift work exposure before and during pregnancy to offspring mental health outcomes.

We consider the longitudinal follow-up of mothers and children, together with detailed information on determinants of maternal lifestyle and social economic status, to be unique strengths of our study.

Limitations should also be mentioned. The timing of the supplemental pregnancy questionnaire led to a small time window of overlap between mothers with available exposure information during pregnancy and children enrolled in GUTS2. Therefore, we had limited power to explore relationships between pregnancy shift work exposure and mental health outcomes in detail.

Further, the self-reported nature of our outcome definition could have contributed to misclassification. While the use of the CESD-10 scale has been validated as a screening tool [37] and the validity of self-reported clinician/physician diagnosed depression has been considered adequate in adults [51], mental health disorders might be under-diagnosed in the general population and especially in the adolescent and young adults [52]. Nevertheless, outcome misclassification was likely non-differential with respect to shift work exposures and maternal chronotype.

Similarly, maternal night shift work exposure is based on self-reports and crude assessments, asking about the duration nurses had worked at least 3 nights per month in addition to working days or evenings. Hence, the exposure may have been misclassified. Most likely the potential misclassification occurred at random and therefore could have biased our results only towards the null.

Also, chronotype was assessed by one sub-item of the Morningness-Eveningness Questionnaire [42] and rather distant in time from the exposure assessment. However, it has been shown that this single-item measure correlates well with the overall Morningness-Eveningness score [53] and that, even though chronotype can change with age, these changes are slow in pace once adulthood is reached [54] and tend to affect everyone equally (i.e., evening chronotypes will always remain more evening types relative to others, even when factoring in the effects of age).

In conclusion, there was no overall association between duration of maternal night shift work before or during pregnancy and offspring depression or anxiety in our study. However, results from stratified analyses indicate that maternal chronotype might play a role in the relationship between maternal night shift work before pregnancy and depression outcomes in their offspring. Future studies with more refined exposure assessments and more detailed longitudinal measures of psychiatric outcomes throughout childhood, adolescence, and adulthood are needed to explore these relationships in more detail.
Acknowledgements Open access funding provided by Medical University of Vienna. We thank the thousands of participants in the Growing Up Today Study 2, as well as their mothers.

Funding This study was supported by Center for Disease Control and Prevention/The National Institute for Occupational Safety and Health grant 5R01OH009803 (PI: Schernhammer E), as well as Grant UM1 CA176726 National Cancer Institute.

\section{Compliance with ethical standards}

Conflict of interest The authors declare that they have no conflict of interest.

Ethical approval All procedures performed in studies involving human participants were in accordance with the ethical standards of the institutional and/or national research committee and with the 1964 Helsinki declaration and its later amendments or comparable ethical standards.

Informed consent Informed consent was obtained from all individual participants included in the study.

Open Access This article is distributed under the terms of the Creative Commons Attribution 4.0 International License (http://creativeco mmons.org/licenses/by/4.0/), which permits unrestricted use, distribution, and reproduction in any medium, provided you give appropriate credit to the original author(s) and the source, provide a link to the Creative Commons license, and indicate if changes were made.

\section{References}

1. Kessler RC, Keller MB, Wittchen HU. The epidemiology of generalized anxiety disorder. Psychiatr Clin N Am. 2001;24(1):19-39.

2. Kessler RC, Ruscio AM, Shear K, et al. Epidemiology of anxiety disorders. Curr Top Behav Neurosci. 2010;2:21-35.

3. Catalano RF, Fagan AA, Gavin LE, et al. Worldwide application of prevention science in adolescent health. Lancet. 2012;379(9826):1653-64.

4. Center for Behavioral Health Statistics and Quality. 2015 National Survey on Drug Use and Health. Detailed Tables (Tables 9.1 to 9.17)

5. Center for Behavioral Health Statistics and Quality. 2015 National Survey on Drug Use and Health. Detailed Tables (Tables 8.1 to 8.87)

6. Association. (2000). Diagnostic and statistical manual of mental disorders (4th ed.)

7. Kessler RC, Bromet EJ. The epidemiology of depression across cultures. Annu Rev Public Health. 2013;34:119-38.

8. Merikangas KR, Nakamura EF, Kessler RC. Epidemiology of mental disorders in children and adolescents. Dialogues Clin. Neurosci. 2009;11(1):7-20.

9. Sandman CA, Davis EP, Buss C, et al. Prenatal programming of human neurological function. Int J Pept. 2011;2011:837596.

10. Kim DR, Bale TL, Epperson CN. Prenatal programming of mental illness: current understanding of relationship and mechanisms. Curr Psychiatry Rep. 2015;17(2):5.

11. Skipper JK, Jung FD, Coffey LC. Nurses and shiftwork: effects on physical health and mental depression. J Adv Nurs. 1990;15(7):835-42. 
12. Luca M, Bellia S, Bellia M, et al. Prevalence of depression and its relationship with work characteristics in a sample of public workers. Neuropsychiatr Dis Treat. 2014;10:519-25.

13. Flo E, Bjorvatn B, Folkard S, et al. A reliability and validity study of the bergen shift work sleep questionnaire in nurses working three-shift rotations. Chronobiol Int. 2012;29(7):937-46.

14. Ferri P, Guadi M, Marcheselli L, et al. The impact of shift work on the psychological and physical health of nurses in a general hospital: a comparison between rotating night shifts and day shifts. Risk Manag Healthc Policy. 2016;9:203-11.

15. Lee A, Myung SK, Cho JJ, et al. Night shift work and risk of depression: meta-analysis of observational studies. J Korean Med Sci. 2017;32(7):1091-6.

16. Pan A, Schernhammer ES, Sun Q, et al. Rotating night shift work and risk of type 2 diabetes: two prospective cohort studies in women. PLoS Med. 2011;8(12):e1001141.

17. Vetter C, Devore EE, Wegrzyn LR, et al. Association between rotating night shift work and risk of coronary heart disease among women. JAMA. 2016;315(16):1726.

18. Wegrzyn LR, Tamimi RM, Rosner BA, et al. Rotating night-shift work and the risk of breast cancer in the nurses' health studies. Am J Epidemiol. 2017;186(5):532-40.

19. Papantoniou K, Castaño-Vinyals G, Espinosa A, et al. Night shift work, chronotype and prostate cancer risk in the MCC-Spain casecontrol study. Int J Cancer. 2015;137(5):1147-57.

20. Vetter C, Devore EE, Ramin CA, et al. Mismatch of sleep and work timing and risk of type 2 diabetes. Diabetes Care. 2015;38(9):1707-13.

21. Bhatti P, Zhang Y, Song X, et al. Nightshift work and genomewide DNA methylation. Chronobiol Int. 2015;32(1):103-12.

22. Zhu Y, Stevens RG, Hoffman AE, et al. Epigenetic impact of long-term shiftwork: pilot evidence from circadian genes and whole-genome methylation analysis. Chronobiol Int. 2011;28(10):852-61.

23. Alasaari JS, Lagus M, Ollila HM, et al. Environmental stress affects DNA methylation of a $\mathrm{CpG}$ rich promoter region of serotonin transporter gene in a nurse cohort. PLoS One. 2012;7(9):e45813.

24. Chen Y-C, Sheen J-M, Tiao M-M, et al. Roles of melatonin in fetal programming in compromised pregnancies. Int J Mol Sci. 2013;14(3):5380-401.

25. Tamura H, Nakamura Y, Terron MP, et al. Melatonin and pregnancy in the human. Reprod Toxicol. 2008;25(3):291-303.

26. Tamura H, Nakamura $Y$, Terron $M$, et al. Melatonin and pregnancy in the human. Reprod Toxicol. 2008;25(3):291-303.

27. Goldman BD. Pattern of melatonin secretion mediates transfer of photoperiod information from mother to fetus in mammals. Sci Signal. 2003;2003(192):pe29-pe29.

28. Torres-Farfan C, Mendez N, Abarzua-Catalan L, et al. A circadian clock entrained by melatonin is ticking in the rat fetal adrenal. Endocrinology. 2011;152(5):1891-900.

29. Ikeda Y, Kumagai H, Skach A, et al. Modulation of circadian glucocorticoid oscillation via adrenal opioid-CXCR29 signaling alters emotional behavior. Cell. 2013;155(6):1323-36.

30. Dietz DM, Laplant Q, Watts EL, et al. Paternal transmission of stress-induced pathologies. Biol Psychiatry. 2011;70(5):408-14.

31. Flory JD, Bierer LM, Yehuda R. Maternal exposure to the holocaust and health complaints in offspring. Dis Markers. 2011;30(2-3):133-9.

32. Yehuda R, Bell A, Bierer LM, et al. Maternal, not paternal, PTSD is related to increased risk for PTSD in offspring of Holocaust survivors. J Psychiatr Res. 2008;42(13):1104-11.

33. Yehuda R, Engel SM, Brand SR, et al. Transgenerational effects of posttraumatic stress disorder in babies of mothers exposed to the world trade center attacks during pregnancy. J Clin Endocrinol Metab. 2005;90(7):4115-8.
34. Straif K, Baan R, Grosse Y, et al. Carcinogenicity of shift-work, painting, and fire-fighting. Lancet Oncol. 2007;8(12):1065-6.

35. Chang S-C, Cassidy A, Willett WC, et al. Dietary flavonoid intake and risk of incident depression in midlife and older women. Am J Clin Nutr. 2016;104(3):704-14.

36. Radloff LS. The CES-D scale. Appl Psychol Meas. 1977;1(3):385-401.

37. Bradley KL, Bagnell AL, Brannen CL. Factorial validity of the center for epidemiological studies depression 10 in adolescents. Issues Ment Health Nurs. 2010;31(6):408-12.

38. Andresen EM, Malmgren JA, Carter WB, et al. Screening for depression in well older adults: evaluation of a short form of the CES-D (Center for Epidemiologic Studies Depression Scale). Am J Prev Med. 1994;10(2):77-84.

39. Willett WC, Sampson L, Stampfer MJ, et al. Reproducibility and validity of a semiquantitative food frequency questionnaire. Am J Epidemiol. 1985;122(1):51-65.

40. McCullough ML, Feskanich D, Stampfer MJ, et al. Diet quality and major chronic disease risk in men and women: moving toward improved dietary guidance. Am J Clin Nutr. 2002;76(6):1261-71.

41. Ainsworth BE, Haskell WL, Leon AS, et al. Compendium of physical activities: classification of energy costs of human physical activities. Med Sci Sports Exerc. 1993;25(1):71-80.

42. Horne JA, Ostberg O. A self-assessment questionnaire to determine morningness-eveningness in human circadian rhythms. Int. J. Chronobiol. 1976;4(2):97-110.

43. United States Census Bureau. Census. 1989.

44. Morris NM, Udry JR. Validation of a self-administered instrument to assess stage of adolescent development. J Youth Adolesc. 1980;9(3):271-80.

45. Chiu W-H, Yang H-J, Kuo P-H. Chronotype preference matters for depression in youth. Chronobiol Int. 2017;34(7):933-41.

46. Vetter C, Chang S-C, Devore EE, et al. Prospective study of chronotype and incident depression among middle- and olderaged women in the Nurses' health study II. J Psychiatr Res. 2018;103:156-60.

47. Strohmaier S, Devore E, Vetter C, et al. Shift work before and during pregnancy and offspring weight outcomes through adolescence. Obesity. 2018;26:1491-500. https://doi.org/10.1002/ oby.22267.

48. Puttonen S, Härmä M, Hublin C. Shift work and cardiovascular disease-pathways from circadian stress to morbidity. Scand J Work Environ Health. 2010;36:96-108.

49. Merikanto I, Kronholm E, Peltonen M, et al. Circadian preference links to depression in general adult population. J Affect Disord. 2015;188:143-8.

50. Alvaro PK, Roberts RM, Harris JK. The independent relationships between insomnia, depression, subtypes of anxiety, and chronotype during adolescence. Sleep Med. 2014;15(8):934-41.

51. Sanchez-Villegas A, Schlatter J, Ortuno F, et al. Validity of a selfreported diagnosis of depression among participants in a cohort study using the structured clinical interview for DSM-IV (SCIDI). BMC Psychiatry. 2008;8:43.

52. Thapar A, Collishaw S, Pine DS, et al. Depression in adolescence. Lancet. 2012;379(9820):1056-67.

53. Zavada A, Gordijn MCM, Beersma DGM, et al. Comparison of the Munich chronotype questionnaire with the HorneOstberg's Morningness-Eveningness score. Chronobiol Int. 2005;22(2):267-78.

54. Roenneberg T, Kuehnle T, Pramstaller PP, et al. A marker for the end of adolescence. Curr Biol. 2004;14(24):R1038-9.

Publisher's Note Springer Nature remains neutral with regard to jurisdictional claims in published maps and institutional affiliations. 